\title{
On Marshall-Olkin Extended Weibull Distribution
}

\author{
Hanan Haj Ahmad \\ Department of Mathematics, University of Hail \\ Hail, KSA \\ hananahm1@yahoo.com \\ Omar M. Bdair* \\ Department of Physics and Basic Sciences \\ Faculty of Engineering Technology, Al-Balqa' Applied University \\ Amman 11134, Jordan \\ bdairmb@yahoo.com \\ M. Ahsanullah \\ Department of Management Sciences, Rider University \\ Lawrenceville, NJ 08648, USA \\ ahsan@rider.edu
}

Received 14 May 2016

Accepted 15 December 2016

In this paper, we introduce a new form of distributions called "Marshall-Olkin Extended Weibull distribution" MOEW. We study some of its structural properties and the shape of its hazard rate function. We provide some methods of point estimation for the unknown parameters and discuss its asymptotic properties. Finally, numerical methods and simulations are used to compare between different methods of estimation, then we study the behavior of parameters under different sample sizes and different values of parameters.

Keywords: Marshall-Olkin distribution; Hazard rate function; Maximum likelihood estimators; Method of moment estimators; Least squares estimators; Percentiles estimators; Simulations; Characterization.

2000 Mathematics Subject Classification: 62H10, 62F10, 78M05, 37M05, 62E10.

\section{Introduction:}

Statistical distributions are commonly used to describe real world phenomena. For this feature, the theory of statistical distribution is widely studied and new distributions are developed. Scientists are interested in developing more flexible statistical distributions, therefore, many generalized classes of distributions have been developed and applied to describe various phenomena. A common advantage of these generalized distributions is that they have more parameters. [16] proposed an important

${ }^{*}$ Corresponding author. 
method of adding a new parameter to an existing distribution. Thus a new family of distributions called Marshall-Olkin (MO) has been defined. This new family of distributions includes the original distribution as a special case and it gives more flexibility to model. [20] introduced a detailed study about the physical interpretation of the MO family.

Weibull distribution is a well known distribution that has many applications in survival analysis and reliability theory. Its importance appears since Weibull distribution considered as a generalization of many other distributions such as: Exponential, Rayleigh and generalized extreme value distributions. Weibull distribution has many nice properties, among others, its hazard rates can be decreasing, constant or increasing. This is one of the attractive properties that make the Weibull distribution so applicable. Weibull distribution has been extensively used over the past decades for modeling data in reliability, engineering and biological studies.

The need for extended form of the Weibull distribution is important in many areas. Some extended forms of Weibull distribution and its applications were referred to [24], [3], [21] and [23]. The extension idea is simply based on adding more parameters to a well-defined distribution provides more flexible new classes of distributions.

Let $\bar{F}(x)=1-F(x)$ denote the survivor function of a continuous random variable $\mathrm{X}$ and $f(x)=$ $\frac{d F(x)}{d x}$ be the probability density function associated with the cumulative distribution function (cdf) $F(x)$, then the MO extended distribution has the survival function

$$
\bar{G}(x)=\frac{\alpha \bar{F}(x)}{F(x)+\alpha \bar{F}(x)} \quad,-\infty<x<\infty, \quad \alpha>0,
$$

where $\bar{\alpha}=1-\alpha$. It is clear that Eq.(1.1) provides a method to obtain a new distribution from an existing one. When $\alpha=1, \bar{G}(x)=\bar{F}(x)$, it is readily seen that $F(x)$ is a special case of $G(x)$.

The probability density function (pdf) corresponding to Eq. (1.1) takes the form

$$
g(x)=\frac{\alpha f(x)}{(1-\bar{\alpha} \bar{F}(x))^{2}} \quad,-\infty<x<\infty, \quad \alpha>0 .
$$

Some special cases of Eq. (1.1) were recently studied in literature. [5] considered Pareto distribution, [18] considered gamma distribution while [6] took lomax distribution. [1] studied the inferences of Marshall-Olkin exponential distribution and [19] studied the moments of order statistics based on Marshall-Olkin exponential distribution.

[8] and [7] studied a new generalization of the geometric and normal distributions using the MO idea. [4] considered the MO extended Weibull distribution with shape and scale parameters, they studied some of its mathematical properties as well as estimation of the model parameters. [17] discussed the Marshall-Olkin extended Weibull family of distributions, some structural properties including moments and order statistics were discussed. [2] considered Marshall-Olkin exponential distribution and he introduced a comparison between different methods of methods of point estimation of the distribution parameters.

In this paper we introduce MO extended Weibull distribution (MOEW). Some structural properties of the density function and hazard rate function are studied. Our goal is to introduce different methods of point estimation for the unknown parameters. Numerical methods are used to make comparison between these methods, also we study the behavior of estimated parameters for different sample sizes and for different parameter(s) values. Basically we compare, the maximum likelihood estimators, moment estimators, estimators based on percentiles and the least square estimators by using simulation techniques. 
The organization of this paper is as follows. In section 2, we introduce MOEW density function and show that it can be expressed as a linear combination of Weibull density function. The hazard rate function for the MOEW is given in section 3. Section 4 describes the methods of point estimation. Some characterization results are presented in section 5, while numerical results and simulation are provided in section 6 .

\section{The probability density function}

Consider Weibull distribution with probability density function (pdf) given by

$$
f(x ; \theta, \beta, \mu)=\frac{\theta}{\beta}\left(\frac{x-\mu}{\beta}\right)^{\theta-1} \exp \left[-\left(\frac{x-\mu}{\beta}\right)^{\theta}\right], \quad x \geq \mu,
$$

where $\theta>0$ is the shape parameter, $\beta>0$ is a scale parameter and $\mu \in \mathbb{R}$ is the location parameter. The cumulative distribution function (cdf) is given by

$$
F(x ; \theta, \beta, \mu)=1-\exp \left[-\left(\frac{x-\mu}{\beta}\right)^{\theta}\right],
$$

and the survival function is $\bar{F}(x ; \theta, \beta, \mu)=\exp \left[-\left(\frac{x-\mu}{\beta}\right)^{\theta}\right]$. Using Eq. (1.1), the survival function of Marshall-Olkin extended Weibull distribution $(M O E W)$ is given by

$$
\bar{G}(x ; \alpha, \theta, \beta, \mu)=\frac{\alpha \exp \left[-\left(\frac{x-\mu}{\beta}\right)^{\theta}\right]}{1-\bar{\alpha} \exp \left[-\left(\frac{x-\mu}{\beta}\right)^{\theta}\right]},
$$

the corresponding pdf of MOEW is

$$
g(x ; \alpha, \theta, \beta, \mu)=\frac{\frac{\alpha \theta}{\beta}\left(\frac{x-\mu}{\beta}\right)^{\theta-1} \exp \left[-\left(\frac{x-\mu}{\beta}\right)^{\theta}\right]}{\left(1-\bar{\alpha} \exp \left[-\left(\frac{x-\mu}{\beta}\right)^{\theta}\right]\right)^{2}},
$$

where $\alpha>0, \theta>0, \beta>0, \mu \in \mathbb{R}$, and $x \geq \mu$.

For $0<\alpha<1$, using binomial expansion of the denominator we can rewrite it as:

$$
\left(1-\bar{\alpha} \exp \left[-\left(\frac{x-\mu}{\beta}\right)^{\theta}\right]\right)^{-2}=\sum_{j=0}^{\infty}(j+1) \bar{\alpha}^{j} \exp \left[-j\left(\frac{x-\mu}{\beta}\right)^{\theta}\right]
$$

where $\Gamma($.$) is the gamma function. Applying the expansion (2.3) in (2.2), yields$

$$
\begin{aligned}
g(x ; \theta, \beta, \mu) & =\alpha \sum_{j=0}^{\infty} \frac{\theta}{\beta^{*}} \bar{\alpha}^{j} \exp \left[-\left(\frac{x-\mu}{\beta^{*}}\right)^{\theta}\right]\left(\frac{x-\mu}{\beta^{*}}\right)^{\theta-1} \\
& =\sum_{j=0}^{\infty} \eta_{j} f_{W E}\left(x ; \alpha, \theta, \beta^{*}, \mu\right),
\end{aligned}
$$

where $f_{W E}\left(x ; \theta, \beta^{*}, \mu\right)$ is the pdf of Weibull distribution with parameters $\theta, \beta^{*}$ and $\mu$, where $\beta^{*}=\frac{\beta}{(j+1)^{1 / \theta}}, \eta_{j}=\alpha \bar{\alpha}^{j}$. 
For $\alpha>1$, we can use similar argument as in Eq. (2.3), and after some algebraic manipulations we can obtain

$$
g(x ; \alpha, \theta, \beta, \mu)=\sum_{j=0}^{\infty} v_{j} f_{W E}\left(x ; \theta, \beta^{*}, \mu\right),
$$

where $v_{j}=\frac{(-1)^{j}}{\alpha(j+1)} \sum_{k=j}^{\infty}(k+1)\left(\begin{array}{l}k \\ j\end{array}\right)\left(1-\frac{1}{\alpha}\right)^{k}$. Note that $\sum_{j=0}^{\infty} \eta_{j}=\sum_{j=0}^{\infty} v_{j}=1$.

Hence the MOEW density function can be expressed as an infinite linear combination of Weibull density function. Therefore expression (2.4) and (2.5) can be used to find many mathematical properties such as the moments of MOEW distribution.

\section{The Hazard Rate Function}

Hazard rate function or failure rate is important in survival analysis as well as reliability theory. The hazard rate function for MOEW is of the form

$$
h(x ; \alpha, \theta, \beta, \mu)=\frac{\frac{\theta}{\beta}\left(\frac{x-\mu}{\beta}\right)^{\theta-1}}{1-\bar{\alpha} \exp \left[-\left(\frac{x-\mu}{\beta}\right)^{\theta}\right]}, \quad \text { for } x \geq \mu .
$$

In order to determine the shape of $h(x ; \alpha, \theta, \beta, \mu)$ it is quite enough to determine the shape of $\log h(x ; \alpha, \theta, \beta, \mu)$, the first derivative of $\log h(x ; \alpha, \theta, \beta, \mu)$ is

$$
\frac{d \log h(x ; \alpha, \theta, \beta, \mu)}{d x}=\frac{s(x)}{(x-\mu)\left(1-\bar{\alpha} \exp \left[-\left(\frac{x-\mu}{\beta}\right)^{\theta}\right]\right)},
$$

where $s(x)=\left(1-\bar{\alpha} \exp \left[-\left(\frac{x-\mu}{\beta}\right)^{\theta}\right]\right)(\theta-1)-\theta \bar{\alpha} \exp \left[-\left(\frac{x-\mu}{\beta}\right)^{\theta}\right]\left(\frac{x-\mu}{\beta}\right)^{\theta}$.

The possible shapes of the hazard rate function are:

1- If $0<\theta<1$, then $s(x)$ is negative for $x \geq \mu$, and hence the hazard function is a decreasing function with $h(\mu)=\infty$ and $h(\infty)=0$.

2- If $\theta>1,0<\alpha<1$, then $s(x)$ has two roots $x_{0}$ and $x_{1}$. The hazard function is increasing on $\left(\mu, x_{0}\right)$, decreasing on $\left(x_{0}, x_{1}\right)$ and increasing on $\left(x_{1}, \infty\right)$ with $h(\mu)=0$ and $h(\infty)=\infty$.

3- If $\theta>1, \alpha>1$, then $\mathrm{s}(\mathrm{x})$ is positive. The hazard rate is increasing with $h(\mu)=0$ and $h(\infty)=$ $\infty$.

\section{Point Estimation}

In this section we introduce four different methods of point estimation for the parameters of MOEW distribution. The asymptotic properties are discussed for the maximum likelihood and method of moment estimators. In section 6, numerical techniques will be used to obtain the estimated values of parameters, then a comparison is done between these estimated values to decide which method is the best.

\subsection{Maximum Likelihood Estimation}

The maximum likelihood estimation (MLE) is widely used in inferential statistics as it has many nice properties such as invariance, consistency and normal approximation property. It depends basically on maximizing the likelihood function of MOEW distribution. Let $X_{1}, X_{2}, \ldots, X_{n}$ be a random 
sample from MOEW, then the log-likelihood function for the vector of parameters $\gamma=(\alpha, \theta, \beta, \mu)$ can be expressed as

$$
\ell(\gamma)=n \log \left(\frac{\alpha \theta}{\beta}\right)-\sum_{i=1}^{n}\left(\frac{x_{i}-\mu}{\beta}\right)^{\theta}+(\theta-1) \sum_{i=1}^{n} \log \left(\frac{x_{i}-\mu}{\beta}\right)-2 \sum_{i=1}^{n} \log \left(1-\bar{\alpha} \exp \left[-\left(\frac{x_{i}-\mu}{\beta}\right)^{\theta}\right]\right) .
$$

From the above log-likelihood equation we compute the derivatives with respect to the parameter vector $\gamma$. Since $x \geq \mu$, then the MLE of the parameter $\mu$ is assumed to be $x_{(1)}$, where $x_{(1)}$ is the first order statistic. So, we need to solve the following three normal equations after equating them to zero:

$$
\begin{aligned}
& f_{1}(\alpha, \theta, \beta)=\frac{\partial \ell(\gamma)}{\partial \alpha}=\frac{n}{\alpha}-2 \sum_{i=1}^{n} \frac{\exp \left[-\left(\frac{x_{i}-\mu}{\beta}\right)^{\theta}\right]}{1-\bar{\alpha} \exp \left[-\left(\frac{x_{i}-\mu}{\beta}\right)^{\theta}\right]} \\
& f_{2}(\alpha, \theta, \beta)=\frac{\partial \ell(\gamma)}{\partial \theta}=\frac{n}{\theta}-\sum_{i=1}^{n} \log \left(\frac{x_{i}-\mu}{\beta}\right)\left(\left(\frac{x_{i}-\mu}{\beta}\right)^{\theta}-1\right)-2 \bar{\alpha} \sum_{i=1}^{n} \frac{\exp \left[-\left(\frac{x_{i}-\mu}{\beta}\right)^{\theta}\right]\left(\frac{x_{i}-\mu}{\beta}\right)^{\theta} \log \left(\frac{x_{i}-\mu}{\beta}\right)}{1-\bar{\alpha} \exp \left[-\left(\frac{x_{i}-\mu}{\beta}\right)^{\theta}\right]} \\
& f_{3}(\alpha, \theta, \beta)=\frac{\partial \ell(\gamma)}{\partial \beta}=-\frac{n \theta}{\beta}+\frac{\theta}{\beta} \sum_{i=1}^{n}\left(\frac{x_{i}-\mu}{\beta}\right)^{\theta}+\frac{2 \theta \bar{\alpha}}{\beta} \sum_{i=1}^{n} \frac{\exp \left[-\left(\frac{x_{i}-\mu}{\beta}\right)^{\theta}\right]\left(\frac{x_{i}-\mu}{\beta}\right)^{\theta}}{1-\bar{\alpha} \exp \left(-\left(\frac{x_{i}-\mu}{\beta}\right)^{\theta}\right)}
\end{aligned}
$$

The solution for the above normal equations is not an easy task as they have not an explicit solution. The MLE's can be obtained numerically using Newton-Raphson method to solve the above normal equation. Many numerical methods were used in the literature to solve such system of nonlinear equations. In this paper, we use the Newton-Raphson method which is one of the mostly used methods. This method depends on the following iterated equation

$$
\left(\begin{array}{c}
\alpha^{(i+1)} \\
\theta^{(i+1)} \\
\beta^{(i+1)}
\end{array}\right)=\left(\begin{array}{c}
\alpha^{(i)} \\
\theta^{(i)} \\
\beta^{(i)}
\end{array}\right)-\left[\begin{array}{lll}
\frac{\partial f_{1}}{\partial \alpha} & \frac{\partial f_{1}}{\partial \theta} & \frac{\partial f_{1}}{\partial \beta} \\
\frac{\partial f_{2}}{\partial \alpha} & \frac{\partial f_{2}}{\partial \theta} & \frac{\partial f_{2}}{\partial \beta} \\
\frac{\partial f_{3}}{\partial \alpha} & \frac{\partial f_{3}}{\partial \theta} & \frac{\partial f_{3}}{\partial \beta}
\end{array}\right]_{(\gamma)} \times\left(\begin{array}{l}
f_{1}(\gamma) \\
f_{2}(\gamma) \\
f_{3}(\gamma)
\end{array}\right) .
$$

The above iterated iterated equation depends on the choice of the initial point $\left(\alpha^{(0)}, \theta^{(0)}, \beta^{(0)}\right)$. We use Mathematica package to produce the new values (iterated values) for $\alpha, \theta$ and $\beta$ based on their past (or initial) values until they converge to their MLE's results.

The normal approximation of the MLE of vector parameter $\gamma$ can be used to construct approximate confidence intervals and for testing hypotheses on the parameters $\alpha, \theta, \beta$ and $\mu$.

From the asymptotic property of the MLE we have that $\sqrt{n}(\widehat{\gamma}-\gamma) \stackrel{d}{\rightarrow} N_{4}\left(0, K^{-1}(\gamma)\right)$, where $K(\gamma)$ is the unit expected information matrix and $K(\gamma)=\lim _{n \rightarrow \infty} \frac{1}{n} I_{n}(\gamma)$. Here $I_{n}(\gamma)$ is the observed information matrix evaluated at $\widehat{\gamma}$. The observed information matrix is given by

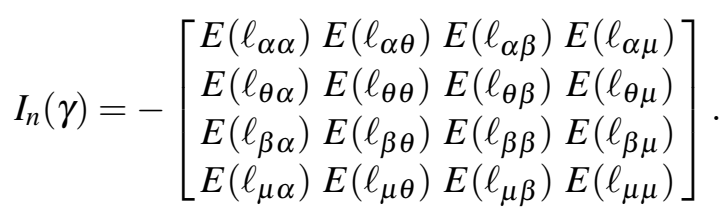

The expected values of the second derivatives can be found by using some methods of integration. Now, without loss of generality, we assume that $\beta=1$. The MLE of $\alpha$, when the other 
parameters are known is obtained by solving the non-linear equation

$$
\frac{n}{\widehat{\alpha}}=2 \sum_{i=1}^{n} \frac{\exp \left[-\left(\frac{x_{i}-\mu}{\beta}\right)^{\theta}\right]}{1-\bar{\alpha} \exp \left[-\left(\frac{x_{i}-\mu}{\beta}\right)^{\theta}\right]} .
$$

The MLE's of $\beta$ and $\theta$ can be obtained directly by maximizing (4.1) with respect to $\beta, \theta$, respectively, given that other parameters are known.

\subsection{Method of Moment Estimation}

In order to use method of moment estimators (MME), it is essential to compute the moments of MOEW distribution. We use the idea of binomial expansion in Eqs. (2.4) and (2.5) in order to obtain the following:

$$
E\left(X^{n}\right)=\sum_{j=0}^{\infty} w_{j} E\left(Y_{j}^{n}\right), w_{j}=\left\{\begin{array}{lc}
\eta_{j} 0<\alpha<1 \\
v_{j} & \alpha>1
\end{array}\right.
$$

where $Y_{j} \sim f_{W E}\left(x ; \theta, \beta^{*}, \mu\right)$. So we divide our work into two cases:

Case (a): $0<\alpha<1$ : The expected value in Eq. (4.3) is written as

$$
E\left(X^{n}\right)=\sum_{j=0}^{\infty} \eta_{j} E\left(Y_{j}^{n}\right) .
$$

The $n^{\text {th }}$ moment for a three parameter Weibull distribution was given by [23]. Using their formula we can rewrite Eq. (4.4) as

$$
E\left(X^{n}\right)=\sum_{j=0}^{\infty} \sum_{i=0}^{n} \eta_{j}\left(\begin{array}{c}
n \\
i
\end{array}\right) \mu^{i}\left(\frac{\beta}{(j+1)^{1 / \theta}}\right)^{(n-i)} \Gamma\left(1+\frac{n-i}{\theta}\right) .
$$

Using the above formula we can find the first four moments. We equate sample moments with the population moments of MOEW to obtain the following four equations:

$$
\begin{gathered}
m_{1}=\bar{X}=E(X)=\mu+\frac{\alpha}{\bar{\alpha}} \beta \Gamma\left(1+\frac{1}{\theta}\right) \text { ploy } \log \left(\frac{1}{\theta}, \bar{\alpha}\right) \\
m_{2}=E\left(X^{2}\right)=\mu^{2}+2 \frac{\alpha}{\bar{\alpha}} \beta \mu \Gamma\left(1+\frac{1}{\theta}\right) \text { ploy } \log \left(\frac{1}{\theta}, \bar{\alpha}\right)+\frac{\alpha}{\bar{\alpha}} \beta^{2} \Gamma\left(1+\frac{2}{\theta}\right) \text { ploy } \log \left(\frac{2}{\theta}, \bar{\alpha}\right), \\
m_{3}=E\left(X^{3}\right)=\mu^{3}+3 \frac{\alpha}{\bar{\alpha}} \beta \mu^{2} \Gamma\left(1+\frac{1}{\theta}\right) \text { ploy } \log \left(\frac{1}{\theta}, \bar{\alpha}\right)+3 \frac{\alpha}{\bar{\alpha}} \beta^{2} \mu \Gamma\left(1+\frac{2}{\theta}\right) \text { ploy } \log \left(\frac{2}{\theta}, \bar{\alpha}\right) \\
+\frac{\alpha}{\bar{\alpha}} \beta^{3} \Gamma\left(1+\frac{3}{\theta}\right) \text { ploy } \log \left(\frac{3}{\theta}, \bar{\alpha}\right), \\
m_{4}=E\left(X^{4}\right)=\mu^{4}+4 \frac{\alpha}{\bar{\alpha}} \beta \mu^{3} \Gamma\left(1+\frac{1}{\theta}\right) \text { ploy } \log \left(\frac{1}{\theta}, \bar{\alpha}\right)+12 \frac{\alpha}{\bar{\alpha}} \beta^{2} \mu^{2} \Gamma\left(1+\frac{2}{\theta}\right) \text { ploy } \log \left(\frac{2}{\theta}, \bar{\alpha}\right) \\
+12 \frac{\alpha}{\bar{\alpha}} \beta^{3} \mu \Gamma\left(1+\frac{3}{\theta}\right) \text { ploylog }\left(\frac{3}{\theta}, \bar{\alpha}\right)+4 \frac{\alpha}{\bar{\alpha}} \beta^{4} \Gamma\left(1+\frac{4}{\theta}\right) \text { ploy } \log \left(\frac{4}{\theta}, \bar{\alpha}\right),
\end{gathered}
$$


where $\operatorname{polylog}(s, z)$ is a special function $\operatorname{Li}_{s}(z)$ which is the polylogarithm function defined by the power series $\operatorname{Li}_{s}(\mathrm{z})=\sum_{k=1}^{\infty} \frac{z^{k}}{k^{s}}$.

Case (b): $\alpha>1$ : In this case the expected value in Eq. (4.3) can be written as

$$
\begin{aligned}
E\left(X^{n}\right) & =\sum_{j=0}^{\infty} v_{j} E\left(Y_{j}^{n}\right)=\sum_{j=0}^{\infty} \sum_{i=0}^{n} v_{j}\left(\begin{array}{c}
n \\
i
\end{array}\right) \mu^{i}\left(\frac{\beta}{(j+1)^{1 / \theta}}\right)^{n-i} \Gamma\left(1+\frac{n-i}{\theta}\right) \\
& =\sum_{j=0}^{\infty} \sum_{i=0}^{n} \sum_{k=j}^{\infty} \frac{(-1)^{j}}{\alpha(j+1)}(k+1)\left(\begin{array}{c}
k \\
j
\end{array}\right)\left(1-\frac{1}{\alpha}\right)^{k}\left(\begin{array}{c}
n \\
i
\end{array}\right) \mu^{i}\left(\frac{\beta}{(j+1)^{1 / \theta}}\right)^{n-i} \Gamma\left(1+\frac{n-i}{\theta}\right) .
\end{aligned}
$$

Using this formula and by applying the same argument used for case $0<\alpha<1$, we can find the first four moments for case $\alpha>1$. In fact, after some mathematical manipulation, we find that the first four moment for this case are the same as the first four moments for case $0<\alpha<1$ given in Eq.'s (4.5)-(4.8), respectively. We use numerical methods to solve the above four equations in order to estimate the needed parameters.

Sometimes population and sample variance can be used to obtain the second moment instead of Eq. (4.6), hence

$$
S^{2}=\sigma^{2}=\frac{\alpha}{\bar{\alpha}} \beta^{2} \Gamma\left(1+\frac{2}{\theta}\right) \text { ploy } \log \left(\frac{2}{\theta}, \bar{\alpha}\right)-\left(\frac{\alpha}{\bar{\alpha}}\right)^{2} \beta^{2} \Gamma^{2}\left(1+\frac{1}{\theta}\right)\left(\text { ploy } \log \left(\frac{1}{\theta}, \bar{\alpha}\right)\right)^{2} .
$$

Now we discuss the asymptotic distribution of the MME's of $\alpha, \theta, \beta$ and $\mu$, let us denote $\gamma=$ $(\alpha, \theta, \beta, \mu)$ and let

$$
\begin{gathered}
f_{1}(\gamma)=\bar{X}-\mu-\frac{\alpha}{\bar{\alpha}} \beta \Gamma\left(1+\frac{1}{\theta}\right) \text { ploy } \log \left(\frac{1}{\theta}, \bar{\alpha}\right), \\
f_{2}(\gamma)=S^{2}-\frac{\alpha}{\bar{\alpha}} \beta^{2} \Gamma\left(1+\frac{2}{\theta}\right) \text { ploy } \log \left(\frac{2}{\theta}, \bar{\alpha}\right)+\left(\frac{\alpha}{\bar{\alpha}}\right)^{2} \beta^{2} \Gamma^{2}\left(1+\frac{1}{\theta}\right)\left(\operatorname{ploy} \log \left(\frac{1}{\theta}, \bar{\alpha}\right)\right)^{2}, \\
f_{3}(\gamma)=E\left(X^{3}\right)-\mu^{3}-3 \frac{\alpha}{\bar{\alpha}} \beta \mu^{2} \Gamma\left(1+\frac{1}{\theta}\right) \text { ploy } \log \left(\frac{1}{\theta}, \bar{\alpha}\right)-3 \frac{\alpha}{\bar{\alpha}} \beta^{2} \mu \Gamma\left(1+\frac{2}{\theta}\right) \text { ploy } \log \left(\frac{2}{\theta}, \bar{\alpha}\right)- \\
\frac{\alpha}{\bar{\alpha}} \beta^{3} \Gamma\left(1+\frac{3}{\theta}\right) p \operatorname{loy} \log \left(\frac{3}{\theta}, \bar{\alpha}\right), \\
f_{4}(\gamma)=E\left(X^{4}\right)-\mu^{4}-4 \frac{\alpha}{\bar{\alpha}} \beta \mu^{3} \Gamma\left(1+\frac{1}{\theta}\right) \text { ploy } \log \left(\frac{1}{\theta}, \bar{\alpha}\right)-12 \frac{\alpha}{\bar{\alpha}} \beta^{2} \mu^{2} \Gamma\left(1+\frac{2}{\theta}\right) \text { ploy } \log \left(\frac{2}{\theta}, \bar{\alpha}\right)- \\
12 \frac{\alpha}{\bar{\alpha}} \beta^{3} \mu \Gamma\left(1+\frac{3}{\theta}\right) \text { ploy } \log \left(\frac{3}{\theta}, \bar{\alpha}\right)-4 \frac{\alpha}{\bar{\alpha}} \beta^{4} \Gamma\left(1+\frac{4}{\theta}\right) \text { ploy } \log \left(\frac{4}{\theta}, \bar{\alpha}\right) .
\end{gathered}
$$

Using Taylor expansion of $f\left(\widehat{\gamma}_{M M}\right)$ about the true value of $\gamma=(\alpha, \theta, \beta, \mu)$, where $f(\gamma)=$ $\left(f_{1}(\gamma), f_{2}(\gamma), f_{3}(\gamma), f_{4}(\gamma)\right)$, we obtain

$$
f\left(\widehat{\gamma}_{M M}\right)-f(\gamma)=\left[\widehat{\alpha}_{M M}-\alpha, \widehat{\theta}_{M M}-\theta, \widehat{\beta}_{M M}-\beta, \widehat{\mu}_{M M}-\mu\right]\left[\begin{array}{l}
\left(\frac{\partial f_{1}}{\partial \alpha}\right)\left(\frac{\partial f_{2}}{\partial \alpha}\right)\left(\frac{\partial f_{3}}{\partial \alpha}\right)\left(\frac{\partial f_{4}}{\partial \alpha}\right) \\
\left(\frac{\partial f_{1}}{\partial \theta}\right)\left(\frac{\partial f_{2}}{\partial \theta}\right)\left(\frac{\partial f_{3}}{\partial \theta}\right)\left(\frac{\partial f_{4}}{\partial \theta}\right) \\
\left(\frac{\partial f_{1}}{\partial \beta}\right)\left(\frac{\partial f_{2}}{\partial \beta}\right)\left(\frac{\partial f_{3}}{\partial \beta}\right)\left(\frac{\partial f_{4}}{\partial \beta}\right) \\
\left(\frac{\partial f_{1}}{\partial \mu}\right)\left(\frac{\partial f_{2}}{\partial \mu}\right)\left(\frac{\partial f_{3}}{\partial \mu}\right)\left(\frac{\partial f_{4}}{\partial \mu}\right)
\end{array}\right]_{\gamma=\bar{\gamma}},
$$


where $\bar{\gamma}$ is a point between $\widehat{\gamma}_{M M}$ and $\gamma$. Its clear that as $n \rightarrow \infty, \widehat{\gamma}_{M M} \rightarrow \gamma$ and $\bar{\gamma} \rightarrow \gamma$. The derivatives mentioned in the above matrix can be obtained simply, let us consider the first one as an example:

$$
\frac{\partial f_{1}}{\partial \alpha}=\frac{\beta \Gamma(1+1 / \theta)}{\bar{\alpha}^{2}}(-\bar{\alpha} \text { poly } \log (-1+1 / \theta, \bar{\alpha})-\alpha \text { poly } \log (1 / \theta, \bar{\alpha})+\alpha \text { poly } \log (-1+1 / \theta, \bar{\alpha})),
$$

where $\operatorname{poly} \Gamma(z)=\psi(z)=\frac{d}{d z} \ln (\Gamma(z))=\frac{\Gamma^{\prime}(z)}{\Gamma(z)}$ and poly $\log ^{(1,0)}(a / \theta, \bar{\alpha})=\frac{-\theta^{2}}{a} \frac{\partial p o l y \log (a / \theta, \bar{\alpha})}{\partial \theta}$. Using central limit theorem we obtain $\sqrt{n}(\bar{X}-E(\bar{X})) \rightarrow N\left(0, \sigma^{2}\right)$ and

$$
\sqrt{n}\left(S^{2}-E\left(S^{2}\right)\right) \rightarrow N\left(0, \frac{(n-1)^{2} m_{4}-(n-1)(n-3) m_{2}^{2}}{n^{3}}\right) .
$$

Therefore

$$
\left[\sqrt{n}(\bar{X}-E(\bar{X})), \sqrt{n}\left(S^{2}-E\left(S^{2}\right)\right)\right] \rightarrow N\left(0,\left[\begin{array}{cc}
\sigma^{2} & \operatorname{Cov}\left(\bar{X}, S^{2}\right) \\
\operatorname{Cov}\left(\bar{X}, S^{2}\right) & \frac{(n-1)^{2} m_{4}-(n-1)(n-3) m_{2}^{2}}{n^{3}}
\end{array}\right],\right.
$$

where $\operatorname{Cov}\left(\bar{X}, S^{2}\right)=\frac{n}{n-1}\left(m_{3}-2 m_{1} m_{2}+m_{1} \sigma^{2}+m_{1}^{3}\right)-m_{1}\left(m_{2}-m_{1}^{2}\right)$.

As a special case, we assume that $\mu=0$ and $\sigma=1$, then

$$
\begin{aligned}
E\left(X^{k}\right) & =\int_{0}^{\infty} x^{k} \alpha \theta x^{\theta-1} \frac{e^{-x^{\theta}}}{\left(1-\bar{\alpha} e^{-x^{\theta}}\right)^{2}} d x \\
& =\sum_{k=0}^{\infty} \int_{0}^{\infty} x^{m} \alpha \theta x^{\theta-1}(k+1)(\bar{\alpha})^{k} e^{-(k+1) x^{\theta}} d x
\end{aligned}
$$

Using the substitution $(k+1) x^{\theta}=t$, we conclude $E\left(X^{k}\right)=\sum_{k=o}^{\infty}\left(\frac{1}{k+1}\right)^{\frac{m}{\theta}} \alpha(\bar{\alpha})^{k} \Gamma\left(1+\frac{m}{\theta}\right)$.

\subsection{Estimators Based on Percentiles}

This method was introduced by [11] and [12], which can be used when the data has a distribution function with closed form. The idea depends on estimating the unknown parameters by fitting straight line to the theoretical percentile points obtained from the distribution function and the sample percentile points. [11] and [12] found that this method can be useful in Weibull and exponential distributions. In this section we use the same technique for the MOEW distribution.

Consider the (cdf) of MOEW distribution

$$
1-G(x ; \gamma)=\frac{\alpha}{\exp \left[\left(\frac{x-\mu}{\beta}\right)^{\theta}\right]-\bar{\alpha}}
$$

where $\gamma=(\alpha, \theta, \beta, \mu)$. Therefore

$$
x=\beta\left[\ln \left(\frac{\alpha}{1-G(x ; \gamma)}+1-\alpha\right)\right]^{1 / \theta}+\mu .
$$

Let $X_{(i)}$ denotes the $i^{t h}$ order statistic from a sample of size n. If $p_{i}$ denotes some estimate of $G\left(x_{(i)} ; \gamma\right)$, then the estimate of $\gamma=(\alpha, \theta, \beta, \mu)$ can be obtained by minimizing

$$
\lambda=\sum_{i=1}^{n}\left(x_{(i)}-\mu-\beta\left[\ln \left(\frac{\alpha}{1-p_{i}}+1-\alpha\right)\right]^{1 / \theta}\right)^{2} .
$$


This method is used by several authors, see for example [2], [9] and [14]. Since Eq. (4.10) is a nonlinear equation it is appropriate to use non-linear optimization technique to find the minimum values of the needed estimators. These estimators are called percentile estimators (PCE's). It is possible to use several $p_{i}$ 's as estimators of $G\left(x_{(i)}\right)$. For example $p_{i}=\frac{i}{n+1}$ is the most used estimator of $G\left(x_{(i)}\right)$, as $\frac{i}{n+1}$ is the expected value of $G\left(x_{(i)}\right)$. In this paper we also use $p_{i}=\frac{i}{n+1}$. Some of the other choices of $p_{i}$ 's are $p_{i}=(i-(3 / 8)) /(n+(1 / 4))$ or $p_{i}=(i-(1 / 2)) / n$, see [15].

We assume that $\alpha$ is known, then the PCE's of $(\theta, \beta, \mu)$ can be obtained by taking partial derivatives with respect to the other three parameters respectively. Let $\pi_{i}=\ln \left(\frac{\alpha}{1-p_{i}}+1-\alpha\right)$, then the three normal equations that should be solved are:

$$
\begin{aligned}
& \frac{\partial \lambda}{\partial \mu}=-2 \sum_{i=1}^{n}\left(x_{(i)}-\mu-\beta\left(\pi_{i}\right)^{1 / \theta}\right) \\
& \frac{\partial \lambda}{\partial \beta}=-2 \sum_{i=1}^{n}\left(x_{(i)}-\mu-\beta\left(\pi_{i}\right)^{1 / \theta}\right)\left(\pi_{i}\right)^{1 / \theta} \\
& \frac{\partial \lambda}{\partial \theta}=2 \frac{\beta}{\theta^{2}} \sum_{i=1}^{n}\left(x_{(i)}-\mu-\beta\left(\pi_{i}\right)^{1 / \theta}\right)\left(\pi_{i}\right)^{1 / \theta} \times \ln \left(\pi_{i}\right) .
\end{aligned}
$$

By solving these normal equations after equating them to zero, we have $\sum_{i=1}^{n} x_{(i)}=n \mu+$ $\beta \sum_{i=1}^{n}\left(\pi_{i}\right)^{1 / \theta}$, hence $\widehat{\mu}_{P C E}=\bar{x}-\frac{\beta}{n} \sum_{i=1}^{n}\left(\pi_{i}\right)^{1 / \theta}$.

It is easy to find $\widehat{\mu}_{P C E}$ when $\beta$ and $\theta$ are known. Now, when $\mu$ and $\theta$ are known and if we denote $G(x)=G(x ; \alpha, \beta, \theta, \mu)$, then

$$
\frac{\alpha}{\exp \left(\frac{x-\mu}{\beta}\right)^{\theta}}-\bar{\alpha}=1-G(x),
$$

which is equivalent to $\beta=\frac{x-\mu}{\left(\ln \left[\frac{\alpha}{1-G(x)}+1-\alpha\right]\right)^{1 / \theta}}$. Therefore, the PCE of $\beta$ can be calculated by minimizing

$$
\sum_{i=1}^{n}\left(\beta-\frac{x_{(i)}-\mu}{\left(\ln \left[\frac{\alpha}{1-p_{i}}+1-\alpha\right]\right)^{1 / \theta}}\right)^{2}
$$

with respect to $\beta$. Hence, the PCE of $\beta$ is given by

$$
\widehat{\beta}_{P C E}=\frac{\sum_{i=1}^{n} \frac{x_{(i)}-\mu}{\left(\ln \left[\frac{\alpha}{1-p_{i}}+1-\alpha\right]\right)^{1 / \theta}}}{n} .
$$

Similarly, when $\mu$ and $\beta$ are known, the PCE of $\theta$ is found to be

$$
\widehat{\theta}_{P C E}=\frac{\sum_{i=1}^{n} \frac{\ln \left(\ln \left[\frac{\alpha}{1-p_{i}}+1-\alpha\right] x_{(i)}\right)}{\ln \left[\frac{x_{(i)}-\mu}{\beta}\right]}}{n} .
$$


Without loss of generality, we can assume $\beta=1$ and $\mu$ and $\theta$ are known. If we denote $G(x)=$ $G(x ; \alpha, 1, \theta, \mu)$ then $\frac{\alpha}{1-G(x)}-\alpha=e^{(x-\mu)^{\theta}}-1$ or equivalently

$$
\alpha=\frac{\left(e^{(x-\mu)^{\theta}}-1\right)(1-G(x))}{G(x)} .
$$

Therefore, the PCE of $\alpha$ can be obtained by minimizing

$$
\sum_{i=1}^{n}\left(\alpha-\frac{\left(e^{\left(x_{(i)}-\mu\right)^{\theta}}-1\right)\left(1-p_{i}\right)}{p_{i}}\right)^{2}
$$

with respect to $\alpha$. In this case the PCE of $\alpha$ is found to be

$$
\widehat{\alpha}_{P C E}=\frac{\sum_{i=1}^{n} \frac{\left(e^{\left(x_{(i)}-\mu\right)^{\theta}}-1\right)\left(1-p_{i}\right)}{p_{i}}}{n} .
$$

Interestingly, all the PCE's estimators have a closed forms when assuming that the other parameters are known.

\subsection{Least Squares Estimators and Weighted Least Squares Estimators}

The method of least squares estimate or regression estimate was first suggested by [22] to estimate the parameters of beta distribution. The method can be described as follows: Suppose $Y_{1}, Y_{2}, \ldots, Y_{n}$ is a random sample of size $\mathrm{n}$ from a distribution function $G($.$) and Y_{(1)}, Y_{(2)}, \ldots, Y_{(n)}$ denote the order statistics of the observed sample. Consider $G\left(Y_{(i)}\right)$ to be the distribution function of the $i^{\text {th }}$ order statistics from the observed sample, then $G\left(Y_{(i)}\right)$ has $U(0,1)$ distribution. Therefore we have

$$
E\left(G\left(Y_{(i)}\right)\right)=\frac{i}{n+1}, \quad \operatorname{Var}\left(G\left(Y_{(i)}\right)\right)=\frac{i(n-i+1)}{(n+1)^{2}(n+2)},
$$

and

$$
\operatorname{Cov}\left(G\left(Y_{(i)}\right), G\left(Y_{(j)}\right)\right)=\frac{i(n-j+1)}{(n+1)^{2}(n+2)}, \quad \text { for } i<j .
$$

See, for example, [10]. The least squares estimators of the unknown parameters can be obtained by minimizing

$$
\sum_{i=1}^{n}\left(G\left(Y_{(i)}\right)-\frac{i}{n+1}\right)^{2}
$$

with respect to the unknown parameters $\alpha, \beta, \theta, \mu$ and hence minimizing

$$
\sum_{i=1}^{n}\left(\frac{e^{\left(\frac{Y_{(i)-\mu}}{\beta}\right)^{\theta}}-1}{e^{\left(\frac{Y_{(i)-\mu}}{\beta}\right)^{\theta}}-(1-\alpha)}-\frac{i}{n+1}\right)^{2} .
$$

Numerically, we can obtain the least squares estimate of the four parameters which are denoted by $\widehat{\alpha}_{L S E}, \widehat{\beta}_{L S E}, \widehat{\theta}_{L S E}, \widehat{\mu}_{L S E}$. 
For the weighted least square estimators of the unknown parameters we need to minimize

$$
\sum_{i=1}^{n} \frac{1}{\operatorname{Var}\left(G\left(Y_{(i)}\right)\right)}\left(G\left(Y_{(i)}\right)-\frac{i}{n+1}\right)^{2} .
$$

Equivalently, the weighted least square estimators can be evaluated by minimizing

$$
\sum_{i=1}^{n} \frac{(n+1)^{2}(n+2)}{i(n-i+1)}\left(\frac{e^{\left(\frac{Y_{(i)-\mu}}{\beta}\right)^{\theta}}-1}{e^{\left(\frac{Y(i)-\mu}{\beta}\right)^{\theta}}-(1-\alpha)}-\frac{i}{n+1}\right)^{2},
$$

to obtain what are denoted by $\widehat{\alpha}_{W L S E}, \widehat{\beta}_{W L S E}, \widehat{\theta}_{W L S E}, \widehat{\mu}_{W L S E}$. Numerical argument are needed to evaluate the so obtained estimators.

\section{Some Characterization Results}

In this section, we present four characterization results based on MOEW distribution. To prove the main results, we need to consider some Lemmas.

Lemma 5.1. Suppose that $X$ is an absolutely continuous random variable and its $c d f F(x)$ with $F(0)=0$ and $F(x)>0$ for all $x>0$. Assume that $f(x)$ is the pdf of $X$ and $f^{\prime}(x)$ exists for all $x>0$. For a continuous function $g(x)$ defined on $0<x<\infty$ with finite $E(g(X))$. If $E(g(X) \mid X \geq$ $x)=h(x) r(x)$, where $h(x)$ is differentiable in $x>0$ and $r(x)=\frac{f(x)}{1-F(x)}$ is the hazard rate, then $f(x)=c \exp \left(-\int_{0}^{x} \frac{g(u)+h^{\prime}(u)}{h(u)} d u\right)$, where $c$ is determined by the condition $\int_{0}^{\infty} f(x) d x=1$.

Proof. Let $h(x)=\frac{\int_{x}^{\infty} g(u) f(u) d u}{f(x)}$, then $\int_{x}^{\infty} g(u) f(u) d u=f(x) h(x)$. Differentiating both sides, we obtain $-g(x) f(x)=f(x) h^{\prime}(x)+f^{\prime}(x) h(x)$. After some simplifications, we have

$$
\frac{f^{\prime}(x)}{f(x)}=-\frac{g(x)+h^{\prime}(x)}{h(x)} .
$$

Integrating both sides of the above equation, we obtain $f(x)=c \exp \left(-\int_{0}^{x} \frac{g(u)+h^{\prime}(u)}{h(u)} d u\right)$ and $c$ is determined by the condition $\int_{0}^{\infty} f(x) d x=1$.

Lemma 5.2. Suppose that $X$ is an absolutely continuous random variable and its cdf $F(x)$ with $F(0)=0$ and $F(x)>0$ for all $x>0$. Assume that the pdf of $X$ is $f(x)$ and $f^{\prime}(x)$ exists for all $x>0$. For a a continuous function $g(x)$ defined on $0<x<\infty$ with finite $E(g(X))$. If $E(g(X) \mid$ $X \leq x)=h(x) \tau(x)$, where $h(x)$ is a differentiable function in $x>0$ and $\tau(x)=\frac{f(x)}{1-F(x)}$, then $f(x)=$ $c \exp \left(-\int_{0}^{x} \frac{g(u)+h^{\prime}(u)}{h(u)} d u\right)$ and $c$ is determined by the condition $\int_{0}^{\infty} f(x) d x=1$.

Proof. We have $h(x)=\frac{\int_{0}^{x} g(u) f(u) d u}{f(x)}$, then $\int_{0}^{x} g(u) f(u) d u=f(x) h(x)$. Differentiating both sides, we obtain $g(x) f(x)=f(x) h^{\prime}(x)+f^{\prime}(x) h(x)$. After some simplifications, we have

$$
\frac{f^{\prime}(x)}{f(x)}=-\frac{g(x)-h^{\prime}(x)}{h(x)} .
$$

Integrating both sides of the above equation, we obtain $f(x)=c \exp \left(-\int_{0}^{x} \frac{g(u)-h^{\prime}(u)}{h(u)} d u\right)$, where $c$ is determined by the condition $\int_{0}^{\infty} f(x) d x=1$. 
Theorem 5.1. Suppose that $X$ is an absolutely continuous random variable and its cdf $F(x)$ with $F(0)=0$ and $F(x)>0$ for all $x>0$. Assume that the $p d f$ of $X$ is $f(x)$ and $f^{\prime}(x)$ exists for all $x>0$ and $E\left(X^{m}\right)$ exists for $m \geq 1$. Then

$$
\begin{aligned}
& E\left(X^{m} \mid X \geq x\right)=h(x) r(x), \text { where } r(x)=\frac{f(x)}{1-F(x)} \text { and } h(x)=\frac{\left(1-\bar{\alpha} e^{-x^{\theta}}\right)^{2}}{c \alpha x^{\theta-1} e^{-x^{\theta}}} \sum_{k=0}^{\infty}\left(\frac{1}{k+1}\right)^{\frac{m}{\theta}} \Gamma_{(k+1) x^{\theta}}^{c}\left(\frac{m+\theta}{\theta}\right) \\
& \text { where } \Gamma_{\alpha}^{c}(\beta)=\int_{\alpha}^{\infty} x^{\beta-1} e^{-x} d x \text { if and only if } f(x)=\frac{c \alpha x^{\theta-1} e^{-x^{\theta}}}{\left(1-\bar{\alpha} e^{-x^{\theta}}\right)^{2}} \text { for } 0<\alpha \leq 1 .
\end{aligned}
$$

Proof. We have

$$
\begin{aligned}
f(x) h(x) & =\int_{x}^{\infty} u^{m} \frac{\theta \alpha u^{\theta-1} e^{-u^{\theta}}}{\left(1-\bar{\alpha} e^{-u^{\theta}}\right)^{2}} d u \\
& =\int_{x}^{\infty} u^{m} \theta \alpha u^{\theta-1} e^{-u^{\theta}} \sum_{k=0}^{\infty}(k+1)(\bar{\alpha})^{k} e^{-k u^{\theta}} d u \\
& =\sum_{k=0}^{\infty}\left(\frac{1}{k+1}\right)^{\frac{m}{\theta}} \Gamma_{(k+1) x^{\theta}}^{c}\left(\frac{m+\theta}{\theta}\right),
\end{aligned}
$$

where $\Gamma_{\alpha}^{c}(\beta)=\int_{\alpha}^{\infty} x^{\beta-1} e^{-x} d x$. Thus

$$
h(x)=\frac{\left(1-\bar{\alpha} e^{-x^{\theta}}\right)^{2}}{c \alpha x^{\theta-1} e^{-x^{\theta}}} \sum_{k=0}^{\infty}\left(\frac{1}{k+1}\right)^{\frac{m}{\theta}} \Gamma_{(k+1) x^{\theta}}^{c}\left(\frac{m+\theta}{\theta}\right) .
$$

Suppose

$$
h(x)=\frac{\left(1-\bar{\alpha} e^{-x^{\theta}}\right)^{2}}{c \alpha x^{\theta-1} e^{-x^{\theta}}} \sum_{k=0}^{\infty}\left(\frac{1}{k+1}\right)^{\frac{m}{\theta}} \Gamma_{(k+1) x^{\theta}}^{c}\left(\frac{m+\theta}{\theta}\right),
$$

then

$$
\frac{x^{m}+h^{\prime}(x)}{h(x)}=\frac{\theta-1}{u}-\theta x^{\theta-1}-\frac{2 \bar{\alpha} \theta x^{\theta-1} \theta e^{-u^{\theta}}}{1-\bar{\alpha} e^{-u^{\theta}}} .
$$

Using Lemma 5.1, we conclude

$$
\frac{f^{\prime}(x)}{f(x)}=\frac{\theta-1}{u}-\theta x^{\theta-1}-\frac{2 \bar{\alpha} \theta x^{\theta-1} \theta e^{-u^{\theta}}}{1-\bar{\alpha} e^{-u^{\theta}}} .
$$

By integrating the above equation, we obtain $f(x)=c \frac{c \alpha x^{\theta-1} e^{-x^{\theta}}}{\left(1-\bar{\alpha} e^{-x^{\theta}}\right)^{2}}$, where $c$ is a constant. Using the condition $\int_{0}^{\infty} f(x) d x=1$, we obtain

$$
f(x)=\frac{c \alpha x^{\theta-1} e^{-x^{\theta}}}{\left(1-\bar{\alpha} e^{-x^{\theta}}\right)^{2}}, 0<\alpha \leq 1 .
$$

Theorem 5.2. Suppose that $X$ is an absolutely continuous random variable and its $c d f F(x)$ with $F(0)=0$ and $F(x)>0$ for all $x>0$. Assume that the pdf of $X$ is $f(x)$ and $f^{\prime}(x)$ exists for all $x>0$ and assume $E\left(X^{m}\right)$ exists for $m \geq 1$. Then $E\left(X^{m} \mid X \leq x\right)=h(x) \tau(x)$, where $\tau(x)=\frac{f(x)}{F(x)}$ and 
$h(x)=\frac{\left(1-\bar{\alpha} e^{-x^{\theta}}\right)^{2}}{c \alpha x^{\theta-1} e^{-x^{\theta}}} \sum_{k=0}^{\infty}\left(\frac{1}{k+1}\right)^{\frac{m}{\theta}} \Gamma_{(k+1) x^{\theta}}\left(\frac{m+\theta}{\theta}\right)$, where $\Gamma_{\alpha}(\beta)=\int_{0}^{\alpha} x^{\beta-1} e^{-x} d x$ if and only if $f(x)=$ $\frac{c \alpha x^{\theta-1} e^{-x^{\theta}}}{\left(1-\bar{\alpha} e^{-x^{\theta}}\right)^{2}}, 0<\alpha \leq 1$.

Proof. We have

$$
\begin{aligned}
f(x) h(x) & =\int_{o}^{x} u^{m} \frac{\theta \alpha u^{\theta-1} e^{-u^{\theta}}}{\left(1-\bar{\alpha} e^{-u^{\theta}}\right)^{2}} d u \\
& =\int_{o}^{x} u^{m} \theta \alpha u^{\theta-1} e^{-u^{\theta}} \sum_{k=0}^{\infty}(k+1)(\bar{\alpha})^{k} e^{-k u^{\theta}} d u \\
& =\sum_{k=0}^{\infty}\left(\frac{1}{k+1}\right)^{\frac{m}{\theta}} \Gamma_{(k+1) x^{\theta}}\left(\frac{m+\theta}{\theta}\right),
\end{aligned}
$$

where $\Gamma_{\alpha}(\beta)=\int_{0}^{\alpha \infty} x^{\beta-1} e^{-x(k+1) x^{\theta}} d x$. Thus

$$
h(x)=\frac{\left(1-\bar{\alpha} e^{-x^{\theta}}\right)^{2}}{c \alpha x^{\theta-1} e^{-x^{\theta}}} \sum_{k=0}^{\infty}\left(\frac{1}{k+1}\right)^{\frac{m}{\theta}} \Gamma_{(k+1) x^{\theta}}\left(\frac{m+\theta}{\theta}\right)
$$

Suppose now that

$$
h(x)=\frac{\left(1-\bar{\alpha} e^{-x^{\theta}}\right)^{2}}{c \alpha x^{\theta-1} e^{-x^{\theta}}} \sum_{k=0}^{\infty}\left(\frac{1}{k+1}\right)^{\frac{m}{\theta}} \Gamma_{(k+1) x^{\theta}}^{c}\left(\frac{m+\theta}{\theta}\right),
$$

then

$$
\frac{x^{m}-h^{\prime}(x)}{h(x)}=\frac{\theta-1}{u}-\theta x^{\theta-1}-\frac{2 \bar{\alpha} \theta x^{\theta-1} \theta e^{-u^{\theta}}}{1-\bar{\alpha} e^{-u^{\theta}}} .
$$

Using Lemma 5.2, we conclude that

$$
\frac{f^{\prime}(x)}{f(x)}=\frac{\theta-1}{u}-\theta x^{\theta-1}-\frac{2 \bar{\alpha} \theta x^{\theta-1} \theta e^{-u^{\theta}}}{1-\bar{\alpha} e^{-u^{\theta}}} .
$$

By integrating the above equation, we obtain $f(x)=c \frac{c \alpha x^{\theta-1} e^{-x^{\theta}}}{\left(1-\bar{\alpha} e^{-x^{\theta}}\right)^{2}}$, where $c$ is a constant. Using the condition $\int_{0}^{\infty} f(x) d x=1$, we obtain

$$
f(x)=\frac{c \alpha x^{\theta-1} e^{-x^{\theta}}}{\left(1-\bar{\alpha} e^{-x^{\theta}}\right)^{2}}, 0<\alpha \leq 1 .
$$

Based on Eq.'s (2.1) and (2.2) and characterization purpose of this distribution, we can assume w.l.o.g $\mu=0$ and $\beta=1$. 


\section{Numerical Computations and Discussions}

In this section, we perform some numerical computations to compare the performances of the different estimators proposed in the previous sections. We perform an extensive simulation study to compare the performances of the different methods in the sense of bias and mean square error (MSE) for different sample sizes and for different parameter values. The generation of the MOEW can be easily obtained through the transformation $X=\mu+\beta\left(\log \left(\frac{\alpha}{(1-U)}+1-\alpha\right)\right)^{1 / \theta}$, where $\mathrm{U}$ is a uniform distribution deviate on $(0,1)$. Mathematica 7 codes are used for generating the MOEW random variables and for solving the non-linear equations as well as for computing the minimization or maximization of the related functions.

Since $\beta$ is the scale parameter and all the estimators are scale invariant, we take $\beta=1$ in all cases considered. We consider various choices of the parameters $\alpha, \theta, \mu$ and sample sizes $n=10,50$ and 100 .

We compute the average relative biases and average relative MSE's over 10,000 runs. This number of runs will give the accuracy in the order $\pm(10,000)^{-5}= \pm 0.01$ (see [13]). Therefore, we report all the results up to three decimal places.

First we consider the estimation of $\alpha$ when other parameters are known. If $\beta, \theta$ and $\mu$ are known, the MLE's and PCE's of $\alpha$ can be obtained directly from (4.2) and (4.16) respectively. The MLE's of $\beta$ and $\theta$ can be obtained directly from Eq. (4.1). The PCE's of $\beta, \theta$ and $\mu$ can be computed directly from Eq. (4.10). The MME's of all parameters can be obtained by solving the non-linear equation (4.5) when other parameters are known. The LSE's and WLSE's can be obtained by minimizing (4.19) and (4.20), respectively, with respect to the needed parameter only. If $\widehat{\alpha}$ is an estimate, then we present the average value of $(\widehat{\alpha} / \alpha)$ and the average MSE of $(\widehat{\alpha} / \alpha)$. The relative average bias and relative average MSE of $(\widehat{\gamma} / \gamma)$, where $\widehat{\gamma}$ is an estimate of $\gamma$, are defined, respectively, as follows: RelativeBias $(\widehat{\gamma} / \gamma)=(1 / k) \sum_{i=1}^{k}\left(\widehat{\gamma}_{i} / \gamma\right)$ and RelativeMSE $(\widehat{\gamma} / \gamma)=$ $\left(1 / k \gamma^{2}\right) \sum_{i=1}^{k}\left(\widehat{\gamma}_{i}-\rho\right)^{2}$, where $\rho=\gamma \operatorname{RelativeBias}(\widehat{\gamma} / \gamma)$ and $k$ is the number of iterations. We are calculated the results for $\alpha=0.2,0.4,0.8$, for $\theta, \mu=0.5,1.0,2.0$ and for $n=10,50$ and 100. The results are presented in 12 different tables. But since the number of tables are very large, we present only 4 tables each of them when $\alpha=0.8$. The other tables are to be given upon request from the corresponding author of this paper. For each method, the average value of $(\widehat{\alpha} / \alpha)$ is given in each box and the corresponding MSE is reported within parenthesis.

It is observed from Table 1 that all of the estimators usually overestimate $\alpha$ for small values of $\alpha,(\alpha \leq 0.4)$. For $\alpha>0.4$, most of the estimators tends to be underestimates $\alpha$ for $\mu>1$. It is also observed that all estimates decrease as the value of $\mu$ increase. One can also observe that for each estimation method, the average relative MSE's decreases as the sample size increases and also as the value of $\alpha$ increases.

It is observed from Table 2 that all of the estimators usually overestimate $\beta$ for all values of $\alpha$ except PCE's that appear to be always underestimate. All estimates tend to be underestimate for large values of $\mu$ and large sample sizes. It is also observed that all estimates decrease as the value of $\mu$ increase. One can also observe that for each estimation method, the average relative MSE's decreases as the sample size increases and also as the values of $\alpha$ and $\mu$ increase.

It is observed from Table 3 that all of the estimators usually underestimate $\theta$ for all values of $\alpha$ except MLE's that tend to be underestimate for $\alpha>0.4$. All estimates tend to be underestimate for large values of $\mu$ and large sample sizes. It is also observed that all estimates decrease as the value 
Table 1. Average relative estimators and average relative MSE's of $\alpha$.

\begin{tabular}{cccccccc}
\hline \multicolumn{7}{c}{$(\alpha, \theta, \mu)$} \\
\hline$n$ & method & $(0.8,0.5,0.5)$ & $(0.8,0.5,1.0)$ & $(0.8,0.5,2.0)$ & $(0.8,1.0,0.5)$ & $(0.8,1.0,1.0)$ & $(0.8,1.0,2.0)$ \\
\hline 10 & MLE & $1.019(0.178)$ & $1.013(0.157)$ & $0.791(0.140)$ & $1.139(0.117)$ & $1.009(0.098)$ & $0.864(0.065)$ \\
& MME & $1.412(0.335)$ & $1.183(0.313)$ & $0.823(0.245)$ & $1.310(0.381)$ & $1.273(0.194)$ & $0.628(0.069)$ \\
& PCE & $1.373(0.614)$ & $1.182(0.322)$ & $0.916(0.217)$ & $1.053(0.299)$ & $0.887(0.273)$ & $0.863(0.232)$ \\
& LSE & $1.993(0.368)$ & $1.008(0.202)$ & $0.817(0.187)$ & $1.267(0.293)$ & $1.234(0.256)$ & $0.872(0.162)$ \\
& WLSE & $1.834(0.293)$ & $1.003(0.192)$ & $0.783(0.150)$ & $1.189(0.284)$ & $1.163(0.245)$ & $0.806(0.153)$ \\
\hline 50 & MLE & $1.013(0.148)$ & $1.007(0.138)$ & $0.762(0.127)$ & $1.112(0.076)$ & $1.002(0.049)$ & $0.802(0.032)$ \\
& MME & $1.387(0.232)$ & $1.095(0.128)$ & $0.887(0.111)$ & $1.816(0.296)$ & $1.649(0.163)$ & $0.802(0.053)$ \\
& PCE & $1.367(0.239)$ & $1.217(0.263)$ & $1.128(0.184)$ & $1.063(0.285)$ & $1.004(0.219)$ & $0.838(0.122)$ \\
& LSE & $1.974(0.297)$ & $1.000(0.194)$ & $0.804(0.152)$ & $1.187(0.249)$ & $1.175(0.218)$ & $0.803(0.136)$ \\
& WLSE & $1.149(0.256)$ & $1.003(0.159)$ & $0.703(0.162)$ & $1.135(0.283)$ & $1.137(0.231)$ & $0.822(0.141)$ \\
\hline 100 & MLE & $1.001(0.063)$ & $0.983(0.082)$ & $0.637(0.071)$ & $1.001(0.064)$ & $0.947(0.038)$ & $0.702(0.029)$ \\
& MME & $1.272(0.141)$ & $1.006(0.121)$ & $0.842(0.109)$ & $1.538(0.234)$ & $1.423(0.119)$ & $0.760(0.027)$ \\
& PCE & $1.193(0.219)$ & $1.137(0.203)$ & $0.814(0.151)$ & $1.017(0.232)$ & $1.003(0.216)$ & $0.745(0.110)$ \\
& LSE & $1.823(0.215)$ & $0.991(0.128)$ & $0.725(0.137)$ & $1.183(0.247)$ & $1.178(0.217)$ & $0.716(0.093)$ \\
& WLSE & $1.115(0.202)$ & $0.981(0.114)$ & $0.601(0.117)$ & $1.113(0.192)$ & $1.092(0.131)$ & $0.625(0.116)$ \\
\hline
\end{tabular}

Table 2. Average relative estimators and average relative MSE's of $\beta$.

\begin{tabular}{cccccccc}
\hline \multicolumn{7}{c}{$(\alpha, \theta, \mu)$} & \\
\hline$n$ & method & $(0.8,0.5,0.5)$ & $(0.8,0.5,1.0)$ & $(0.8,0.5,2.0)$ & $(0.8,1.0,0.5)$ & $(0.8,1.0,1.0)$ & $(0.8,1.0,2.0)$ \\
\hline 10 & MLE & $1.113(0.112)$ & $1.058(0.092)$ & $1.013(0.087)$ & $1.127(0.063)$ & $1.125(0.061)$ & $1.011(0.052)$ \\
& MME & $1.036(0.116)$ & $1.028(0.101)$ & $1.013(0.073)$ & $1.030(0.071)$ & $1.026(0.062)$ & $1.015(0.058)$ \\
& PCE & $0.923(0.173)$ & $0.921(0.102)$ & $0.893(0.065)$ & $0.937(0.063)$ & $0.924(0.057)$ & $0.910(0.051)$ \\
& LSE & $1.750(0.191)$ & $1.028(0.125)$ & $1.018(0.111)$ & $1.119(0.092)$ & $1.104(0.089)$ & $1.101(0.082)$ \\
& WLSE & $1.582(0.110)$ & $1.013(0.123)$ & $0.812(0.102)$ & $1.144(0.087)$ & $1.113(0.081)$ & $1.103(0.077)$ \\
\hline 50 & MLE & $1.083(0.103)$ & $1.081(0.092)$ & $1.072(0.082)$ & $1.062(0.068)$ & $1.059(0.065)$ & $1.004(0.056)$ \\
& MME & $1.007(0.105)$ & $1.004(0.101)$ & $1.001(0.076)$ & $1.004(0.062)$ & $1.000(0.057)$ & $0.983(0.054)$ \\
& PCE & $0.914(0.101)$ & $0.912(0.097)$ & $0.907(0.054)$ & $0.942(0.063)$ & $0.936(0.060)$ & $0.918(0.045)$ \\
& LSE & $1.612(0.165)$ & $1.003(0.108)$ & $0.893(0.093)$ & $1.152(0.088)$ & $1.117(0.081)$ & $0.938(0.078)$ \\
& WLSE & $1.410(0.097)$ & $1.001(0.095)$ & $0.592(0.081)$ & $1.365(0.068)$ & $1.187(0.062)$ & $0.901(0.057)$ \\
\hline 100 & MLE & $1.055(0.087)$ & $1.012(0.085)$ & $1.007(0.081)$ & $1.046(0.055)$ & $1.031(0.043)$ & $0.973(0.027)$ \\
& MME & $1.002(0.089)$ & $0.990(0.079)$ & $0.983(0.067)$ & $1.000(0.064)$ & $0.992(0.061)$ & $0.912(0.043)$ \\
& PCE & $0.908(0.094)$ & $0.902(0.088)$ & $0.893(0.052)$ & $0.923(0.051)$ & $0.917(0.042)$ & $0.901(0.050)$ \\
& LSE & $1.519(0.097)$ & $1.002(0.087)$ & $0.881(0.082)$ & $1.115(0.071)$ & $1.109(0.067)$ & $0.971(0.051)$ \\
& WLSE & $1.112(0.081)$ & $1.001(0.073)$ & $0.516(0.063)$ & $1.156(0.061)$ & $1.101(0.054)$ & $0.524(0.040)$ \\
\hline
\end{tabular}

of $\mu$ increases. One can also observe that for each estimation method, the average relative MSE's decreases as the sample size increases and also as the values of $\alpha$ and $\mu$ increase.

It is observed from Table 4 that most of the estimators usually underestimate $\mu$ for all values of $\alpha$ especially for large values of $\mu$. All estimates tend to be underestimate for large values of $\mu$ and large sample sizes except MLE's that appear to be always overestimate. It is also observed that all estimates decrease as the values of $\mu$ and $\alpha$ increase but they increase as the sample sizes increase. One can also observe that for each estimation method, the average relative MSE's decreases as the sample size increases and also as the values of $\alpha$ and $\mu$ increase.

The MLE's provide the best results for all sample sizes. The WLSE's work better than the LSE's for all sample sizes and all values of $\alpha$. In the context of computational issues, the MLE's, MME's 
Table 3. Average relative estimators and average relative MSE's of $\theta$.

\begin{tabular}{cccccccc}
\hline \multicolumn{7}{c}{$(\alpha, \theta, \mu)$} & \\
\hline$n$ & method & $(0.8,0.5,0.5)$ & $(0.8,0.5,1.0)$ & $(0.8,0.5,2.0)$ & $(0.8,1.0,0.5)$ & $(0.8,1.0,1.0)$ & $(0.8,1.0,2.0)$ \\
\hline 10 & MLE & $0.984(0.092)$ & $0.980(0.059)$ & $0.975(0.056)$ & $0.992(0.061)$ & $0.981(0.058)$ & $0.971(0.053)$ \\
& MME & $1.190(0.039)$ & $0.997(0.036)$ & $0.812(0.028)$ & $0.601(0.021)$ & $0.595(0.019)$ & $0.589(0.010)$ \\
& PCE & $1.112(0.172)$ & $0.912(0.070)$ & $0.892(0.067)$ & $0.725(0.072)$ & $0.506(0.063)$ & $0.489(0.071)$ \\
& LSE & $1.169(0.061)$ & $0.987(0.021)$ & $0.701(0.015)$ & $0.517(0.018)$ & $0.349(0.011)$ & $0.301(0.005)$ \\
& WLSE & $1.111(0.039)$ & $1.001(0.009)$ & $0.699(0.004)$ & $0.505(0.018)$ & $0.322(0.009)$ & $0.295(0.005)$ \\
\hline 50 & MLE & $0.979(0.061)$ & $0.973(0.053)$ & $0.966(0.050)$ & $0.981(0.050)$ & $0.965(0.048)$ & $0.958(0.042)$ \\
& MME & $1.065(0.65)$ & $0.982(0.060)$ & $0.805(0.058)$ & $0.585(0.041)$ & $0.581(0.009)$ & $0.543(0.006)$ \\
& PCE & $0.884(0.059)$ & $0.865(0.056)$ & $0.851(0.055)$ & $0.713(0.066)$ & $0.501(0.059)$ & $0.476(0.053)$ \\
& LSE & $1.089(0.054)$ & $0.953(0.047)$ & $0.664(0.012)$ & $0.482(0.015)$ & $0.340(0.011)$ & $0.293(0.004)$ \\
& WLSE & $1.003(0.027)$ & $0.996(0.023)$ & $0.639(0.010)$ & $0.486(0.012)$ & $0.317(0.005)$ & $0.281(0.004)$ \\
\hline 100 & MLE & $0.972(0.054)$ & $0.968(0.043)$ & $0.957(0.043)$ & $0.976(0.046)$ & $0.961(0.041)$ & $0.941(0.032)$ \\
& MME & $1.005(0.056)$ & $0.971(0.057)$ & $0.843(0.051)$ & $0.611(0.041)$ & $0.572(0.009)$ & $0.523(0.005)$ \\
& PCE & $0.872(0.066)$ & $0.823(0.059)$ & $0.739(0.052)$ & $0.705(0.051)$ & $0.490(0.046)$ & $0.431(0.023)$ \\
& LSE & $0.983(0.047)$ & $0.951(0.042)$ & $0.610(0.017)$ & $0.478(0.015)$ & $0.332(0.009)$ & $0.284(0.006)$ \\
& WLSE & $0.983(0.022)$ & $0.980(0.019)$ & $0.601(0.011)$ & $0.473(0.015)$ & $0.314(0.004)$ & $0.275(0.004)$ \\
\hline
\end{tabular}

Table 4. Average relative estimators and average relative MSE's of $\mu$.

\begin{tabular}{|c|c|c|c|c|c|c|c|}
\hline \multicolumn{8}{|c|}{$(\alpha, \theta, \mu)$} \\
\hline$n$ & method & $(0.8,0.5,0.5)$ & $(0.8,0.5,1.0)$ & $(0.8,0.5,2.0)$ & $(0.8,1.0,0.5)$ & $(0.8,1.0,1.0)$ & $(0.8,1.0,2.0)$ \\
\hline \multirow[t]{5}{*}{10} & MLE & $1.032(0.061)$ & $1.016(0.048)$ & $1.009(0.034)$ & $1.035(0.041)$ & $1.018(0.025)$ & $1.007(0.019)$ \\
\hline & MME & $1.094(0.125)$ & $1.037(0.127)$ & $0.746(0.119)$ & $0.589(0.276)$ & $0.541(0.255)$ & $0.526(0.212)$ \\
\hline & PCE & $0.903(0.107)$ & $0.892(0.053)$ & $0.884(0.049)$ & $0.915(0.048)$ & $0.904(0.041)$ & $0.897(0.036)$ \\
\hline & LSE & $1.093(0.099)$ & $0.902(0.046)$ & $0.783(0.039)$ & $0.703(0.031)$ & $0.628(0.018)$ & $0.614(0.012)$ \\
\hline & WLSE & $1.087(0.077)$ & $0.931(0.052)$ & $0.816(0.030)$ & $0.707(0.030)$ & $0.663(0.018)$ & $0.628(0.016)$ \\
\hline \multirow[t]{5}{*}{50} & MLE & $1.034(0.059)$ & $1.024(0.039)$ & $1.019(0.032)$ & $1.045(0.039)$ & $1.022(0.031)$ & $1.017(0.017)$ \\
\hline & MME & $1.107(0.119)$ & $1.045(0.112)$ & $0.815(0.109)$ & $0.584(0.222)$ & $0.572(0.212)$ & $0.547(0.180)$ \\
\hline & PCE & $0.937(0.048)$ & $0.917(0.045)$ & $0.892(0.041)$ & $0.937(0.045)$ & $0.916(0.037)$ & $0.901(0.029)$ \\
\hline & LSE & $1.142(0.023)$ & $0.964(0.021)$ & $0.790(0.009)$ & $0.734(0.006)$ & $0.680(0.004)$ & $0.636(0.003)$ \\
\hline & WLSE & $1.092(0.020)$ & $1.072(0.018)$ & $0.868(0.009)$ & $0.789(0.005)$ & $0.733(0.003)$ & $0.701(0.002)$ \\
\hline \multirow[t]{5}{*}{100} & MLE & $1.082(0.017)$ & $1.076(0.015)$ & $1.031(0.009)$ & $1.052(0.012)$ & $1.041(0.007)$ & $1.032(0.002)$ \\
\hline & MME & $1.127(0.107)$ & $1.119(0.093)$ & $0.870(0.082)$ & $0.612(0.111)$ & $0.594(0.094)$ & $0.567(0.087)$ \\
\hline & PCE & $0.973(0.045)$ & $0.958(0.041)$ & $0.919(0.033)$ & $0.942(0.030)$ & $0.927(0.022)$ & $0.914(0.019)$ \\
\hline & LSE & $1.192(0.021)$ & $1.187(0.009)$ & $0.901(0.007)$ & $0.782(0.006)$ & $0.709(0.004)$ & $0.683(0.002)$ \\
\hline & WLSE & $1.099(0.015)$ & $1.085(0.007)$ & $0.907(0.004)$ & $0.870(0.004)$ & $0.749(0.002)$ & $0.721(0.001)$ \\
\hline
\end{tabular}

and PCE's could not be easily implemented since they involve non-linear equations. The LSE's and WLSE's involve non-linear functions that should be minimized.

\section{Acknowledgments}

The authors would like to thank the anonymous referees for their very constructive suggestions that contributed to the improvement of this version of the paper. 


\section{References}

[1] M. Ahsanullah, Some Inferences on Marshall-Olkin Exponential Distribution, Journal of Applied Statistical Science 20(4) (2012) 357-363.

[2] O.M. Bdair, Different Methods of Estimation for Marshall Olkin Exponential Distribution, Journal of Applied Statistical Science 19 (2) (2012) 13-29.

[3] G.M. Cordeiro, E.M.M. Ortega, and S. Nadarajah, The Kumaraswamy Weibull Distribution with Application to Failure Data, Journal of the Franklin Institute 347(8) (2010) 1399-1429.

[4] G.M. Cordeiro, A.J. Lemonte, On the Marshall-Olkin Extended Weibull Distribution, Statistical Papers 54(2) (2013) 333-353

[5] M.E. Ghitany, Marshall-Olkin Extended Pareto Distribution and Its Application, International Journal of Applied Mathematics 18 (2005) 17-32.

[6] M.E. Ghitany, F.A. Al-Awadhi and L.A. Alkhalfan, Marshall-Olkin Extended Lomax Distribution and Its Application to Censored Data, Communications in Statistics - Theory and Methods 36(10) (2007) $1855-1866$.

[7] V.J. García, E. Gómez-Déniz and F.J. Vázquez-Polo, A New Skew Generalization of the Normal Distribution: Properties and Applications, Computational Statistics and Data Analysis 54(8) (2010) 20212034.

[8] E. Gómez-Déniz, Another Generalization of the Geometric Distribution, Test 19(2) (2010) 399-415.

[9] R. Gupta and D. Kundu, Generalized Exponential Distribution: Different Method of Estimations, Journal of Statistical Computation and Simulation 69(4) (2001) 315-337.

[10] N.L. Johnson, S. Kotz and N. Balakrishnan, Continuous Univariate Distribution, Vol. 1, 2nd Ed., (Wiley, New York, 1994).

[11] J.H.K. Kao, Computer Methods for Estimating Weibull Parameters in Reliability Studies, IRE Transactions on Reliability and Quality Control 13 (1958) 15-22.

[12] J.H.K. Kao, A Graphical Estimation of Mixed Weibull Parameters in Life Testing Electron Tube, Technometrics 1(4) (1959) 389-407.

[13] Z.A. Karian and E.J. Dudewicz, Modern Statistical Systems and GPSS Simulations, 2nd edition, (CRC Press, Florida, 1998).

[14] D. Kundu, and M. Raqab, Generalized Rayleigh Distribution: Different Methods of Estimations, Computational Statistics \& Data Analysis 49(1) (2005) 187-200.

[15] N.R. Mann, R.E. Schafer and N.D. Singpurwalla, Methods for Statistical Analysis of Reliability and Life Data, (Wiley, New York, 1974).

[16] A.W. Marshall and I. Olkin, A New Method for Adding a Parameter to a Family of Distributions with Application to the Exponential and Weibull Families, Biometrika 84(3) (1997) 641-652.

[17] M. Neto, M. Bourguignon, L. Zea, A. Nascimento and G.M. Cordeiro, The Marshall-Olkin Extended Weibull Family of Distributions, Journal of Statistical Distributions and Applications 1(9) (2014).

[18] M.M. Ristić, K.K. Jose and J. Ancy, A Marshall-Olkin Gamma Distribution and Minification Process, Stress Anxiety Res Soc 11 (2007) 107-117.

[19] M.S. Salah, M.Z. Raqab and M. Ahsanullah, Marshall-Olkin Exponential Distribution: Moments of Order Statistics, Journal of Applied Statistical Science 17(1) (2010) 91-102.

[20] P.G. Sankaran and K. Jayakumar, On Proportional Odds Model, Statistical Papers 49(4) (2008) 779789.

[21] G.O. Silva, E.M.M. Ortega and G.M. Cordeiro, The Beta Modified Weibull Distribution, Lifetime Data Analysis 16(3) (2010) 409-430.

[22] J.J. Swain, S. Venkatraman and J.R. Wilson, Least Squares Estimation of Distribution Function in Johnson's Translation System, Journal of Statistical Computation and Simulation 29(4) (1988) 271297.

[23] M. Teimouri and A. Gupta, On the Three-Parameter Weibull Distribution Shape Parameter Estimation, Journal of Data Science 11 (2013) 403-414.

[24] M. Xie, Y. Tang and T.N. Goh, A Modified Weibull Extension with Bathtub-Shaped Failure Rate Function, Reliability Engineering \& System Safety 76(3) (2002) 279-285. 